\title{
Video Article \\ Bioelectric Analyses of an Osseointegrated Intelligent Implant Design System for Amputees
}

\author{
Brad M. Isaacson ${ }^{1,2}$, Jeroen G. Stinstra ${ }^{3}$, Rob S. MacLeod ${ }^{2,3}$, Joseph B. Webster ${ }^{1,4}$, James P. Beck ${ }^{1,5}$, Roy D. Bloebaum ${ }^{1,2,5}$ \\ ${ }^{1}$ Department of Veteran Affairs \\ ${ }^{2}$ Department of Bioengineering, University of Utah \\ ${ }^{3}$ Scientific Computing and Imaging Institute, University of Utah \\ ${ }^{4}$ Department of Physical Medicine and Rehabilitation, University of Utah \\ ${ }^{5}$ Department of Orthopaedics, University of Utah
}

Correspondence to: Brad M. Isaacson at brad.isaacson@utah.edu

URL: https://www.jove.com/video/1237

DOI: doi:10.3791/1237

Keywords: Medicine, Issue 29, Osseointegration, Electrical Stimulation, Osteogenesis, Amputation, Percutaneous

Date Published: 7/15/2009

Citation: Isaacson, B.M., Stinstra, J.G., MacLeod, R.S., Webster, J.B., Beck, J.P., Bloebaum, R.D. Bioelectric Analyses of an Osseointegrated Intelligent Implant Design System for Amputees. J. Vis. Exp. (29), e1237, doi:10.3791/1237 (2009).

\section{Abstract}

The projected number of American amputees is expected to rise to 3.6 million by 2050. Many of these individuals depend on artificial limbs to perform routine activities, but prosthetic suspensions using traditional socket technology can prove to be cumbersome and uncomfortable for a person with limb loss. Moreover, for those with high proximal amputations, limited residual limb length may prevent exoprosthesis attachment all together. Osseointegrated implant technology is a novel operative procedure which allows firm skeletal attachment between the host bone and an implant. Preliminary results in European amputees with osseointegrated implants have shown improved clinical outcomes by allowing direct transfer of loads to the bone-implant interface. Despite the apparent advantages of osseointegration over socket technology, the current rehabilitation procedures require long periods of restrictive load bearing prior which may be reduced with expedited skeletal attachment via electrical stimulation. The goal of the osseointegrated intelligent implant design (OIID) system is to make the implant part of an electrical system to accelerate skeletal attachment and help prevent periprosthetic infection. To determine optimal electrode size and placement, we initiated proof of concept with computational modeling of the electric fields and current densities that arise during electrical stimulation of amputee residual limbs. In order to provide insure patient safety, subjects with retrospective computed tomography scans were selected and three dimensional reconstructions were created using customized software programs to ensure anatomical accuracy (Seg3D and SCIRun) in an IRB and HIPAA approved study. These software packages supported the development of patient specific models and allowed for interactive manipulation of electrode position and size. Preliminary results indicate that electric fields and current densities can be generated at the implant interface to achieve the homogenous electric field distributions required to induce osteoblast migration, enhance skeletal fixation and may help prevent periprosthetic infections. Based on the electrode configurations experimented with in the model, an external two band configuration will be advocated in the future.

\section{Video Link}

The video component of this article can be found at https://www.jove.com/video/1237/

\section{Protocol}

\section{Part 1: Using Computed Tomography (CT) Scans for Amputee Reconstruction}

1. Retrospective CT scans were collected from the University of Utah and Department of Veteran Affairs hospitals after obtaining IRB and HIPAA approval.

2. CT scans were selected because they allow clear distinction between tissue types based on x-ray absorbency

3. CTs were manually inspected and included in the study based on the absence of metal implants to prevent image artifact.

\section{Part 2: Model Generation with Seg3D}

1. Files were downloaded as Dicom images and loaded into Seg3D (version 1.11.0, software.sci.utah.edu) as a new volume.

2. A median filter was used to smooth the imported volumes prior to determining geometrically defined tissue structures.

3. The tissue boundaries of the bone, bone marrow, organs and adipose tissue were generated by thresholding the CT files interactively (Figure 1). 

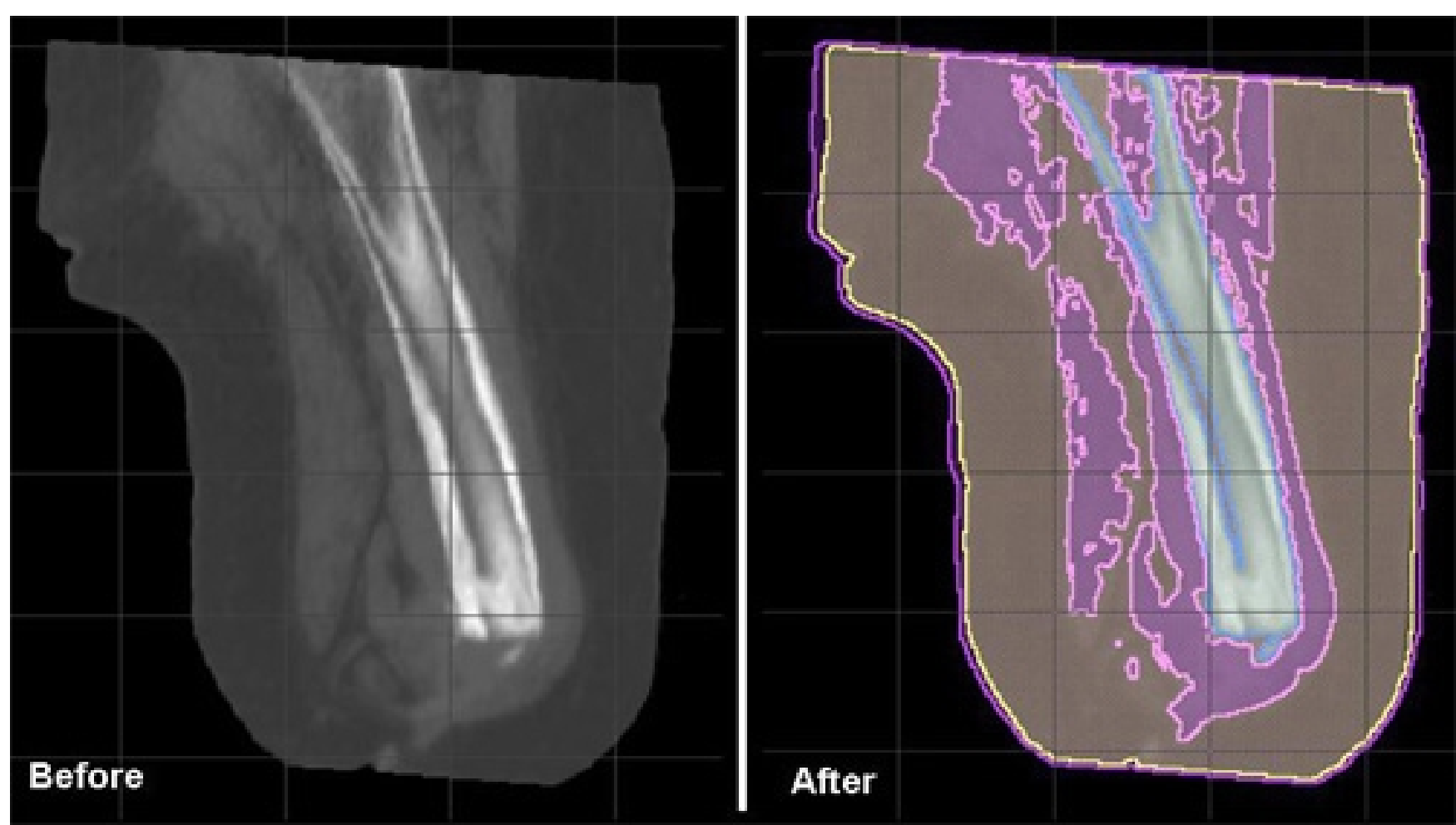

Figure 1: A sagittal cross section of an amputee residual limb thresholded and separated into specific tissue types.

4. The musculature was obtained by manually setting seed points inside the thresholded muscle tissue and using a confidence connected filter to find all the tissue connected to the seed points. This step eliminated erroneous tissues which may have been grouped together with the muscle based on similar absorbency from CTs.

5. The skin, which was impossible to discern reliably from the CT images, was generated by dilating the outermost tissue 2 millimeters based on average skin thickness to produce a layer of homogeneous thickness that surrounded the full model ${ }^{1}$.

6. Segmentations were manually inspected, corrected to ensure accuracy and combined in a hierarchy into a single label map required for finite element analysis (Figure 1). 


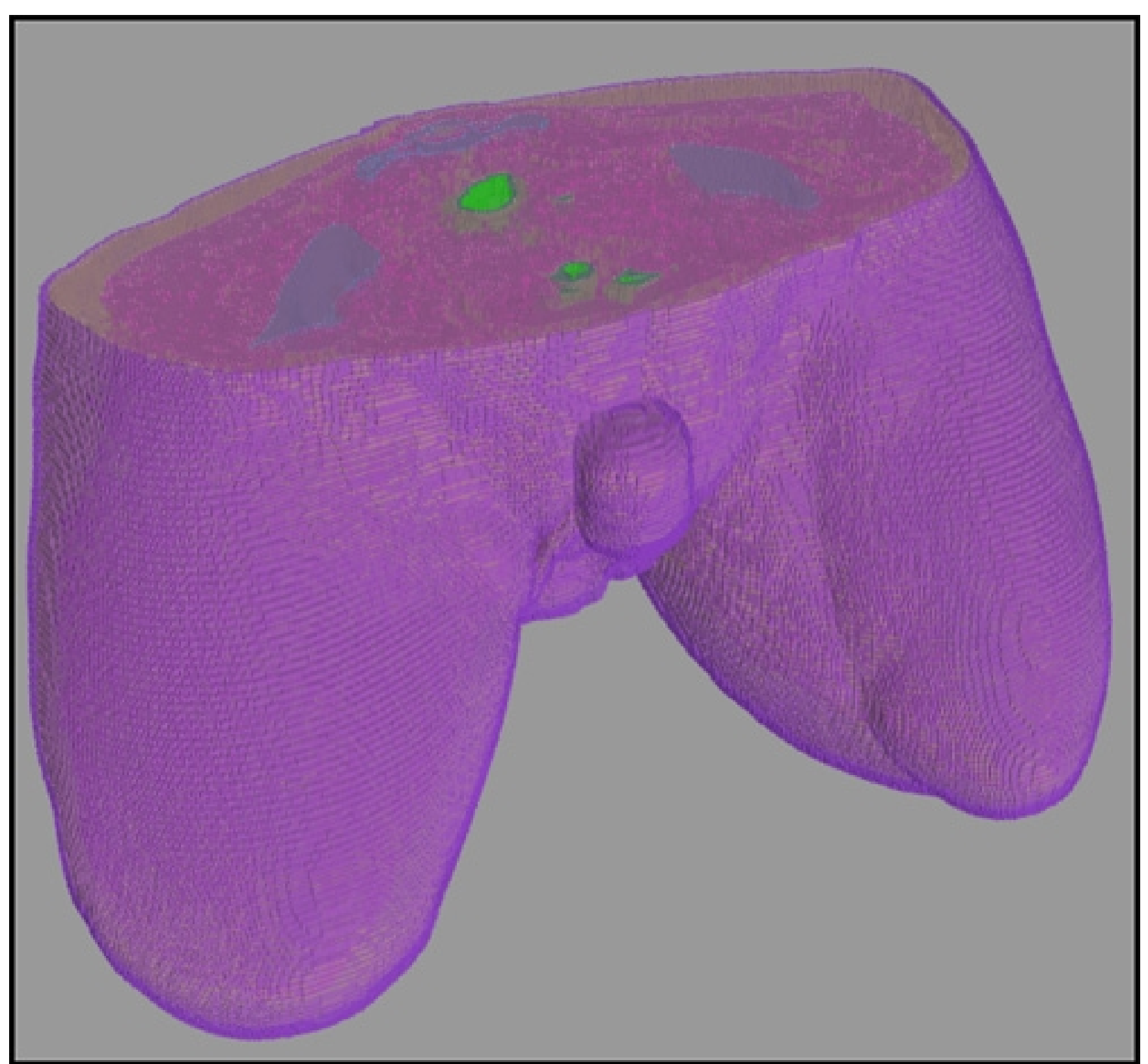

Figure 2: Representative hierarchical model of a bilateral amputee created with Seg3D.

\section{Part 3: Preparation for Finite Element Analysis}

1. A $10 \mathrm{~cm}$ implant was designed in Matlab to serve as the implanted orthopedic device and cathode for electrical stimulation and imported into SCIRun (version 4.0, software.sci.utah.edu).

\section{Part 4: Electrode Placement \& Design}

1. SCIRun was utilized for electrode design because it supports interactive electrode placement and simulation.

2. A network was created and modules organized with specific functions to generate the mesh (Figure 3). Modules were important for defining boundary conditions, tissue conductivities, mesh refinements, generating Matlab histograms, recording field data, etc (Table 1). 


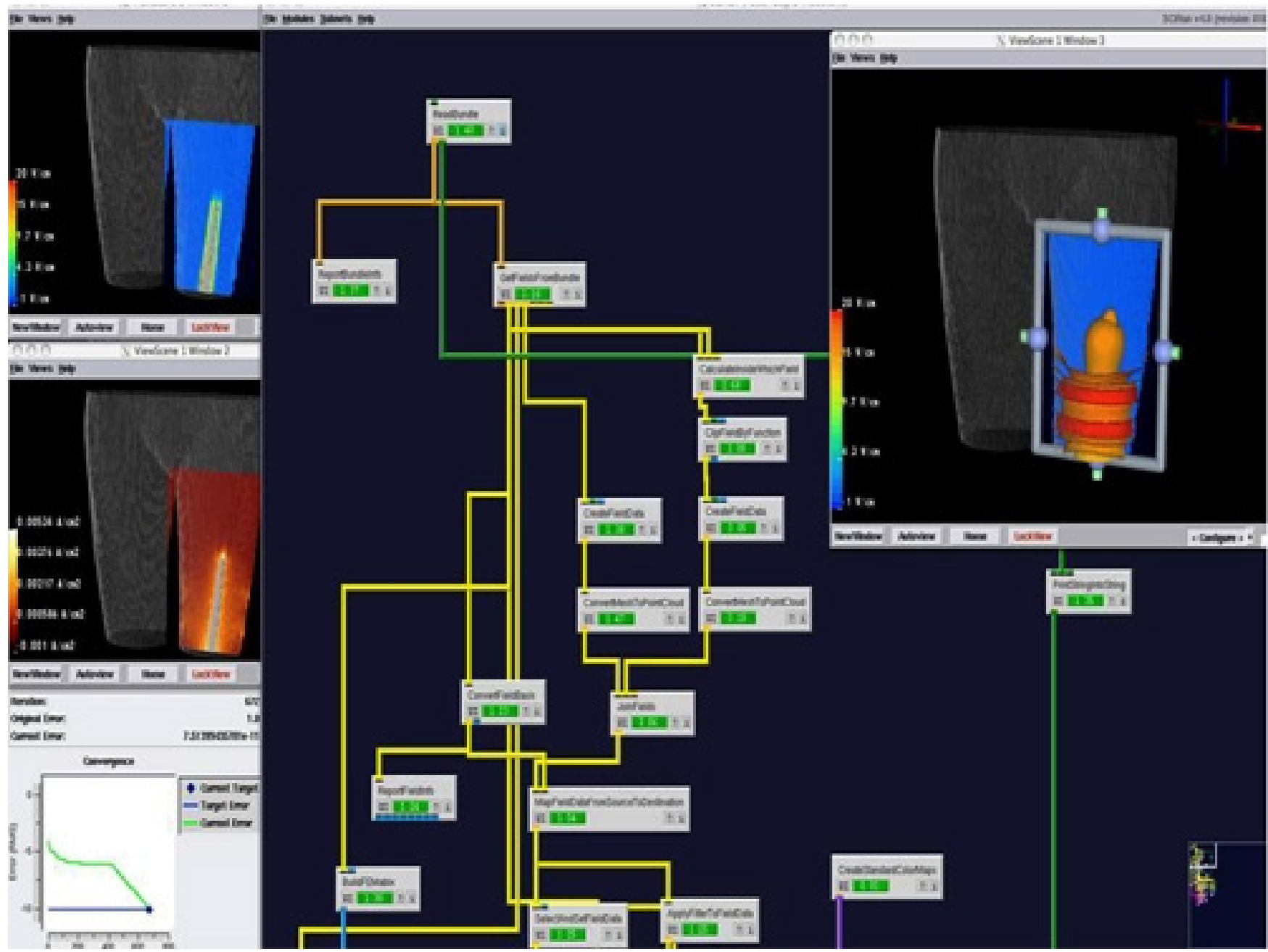

Figure 3: Representative network image from a pilot study using a two band external electrode configuration.

\begin{tabular}{|l|l|}
\hline Table 1 & \multicolumn{2}{|l|}{} \\
\hline Conductivities Assigned to Segmented Tissues & Conductivities [S/m] \\
\hline Tissue Type & 0.22 \\
\hline Organ & 0.26 \\
\hline Skin & 0.09 \\
\hline Adipose & 0.25 \\
\hline Muscle & 0.02 \\
\hline Cortical Bone & 0.07 \\
\hline Bone Marrow & \\
\hline
\end{tabular}

3. The configurations for the electrodes consisted of a one patch electrode, two patch electrodes, one continuous band and two continuous bands.

4. External electrode bands were applied to the residual limb of the models generated from patient CT scans and were $1.6 \mathrm{~cm}$ in thickness.

5. Electrode patches were placed as a strip covering approximately half the diameter of the residual limb and were $3 \mathrm{~cm}$ in thickness.

6 . The inner cortical implant which represented the osseointegrated implant was set to endosteal diameter to allow for perfect implant fit and fill ${ }^{2}$.

\section{Part 5: Finite Element Analysis}

1. The simulations were generated assuming that the electric metrics could be calculated using a quasi-static approach with no time dependency.

2. The model was computed by solving Laplace's equation for each tissue type generated from the Seg3D segmentations.

3. The boundary conditions were formed by the electrodes that injected currents and the guideline that current remained within the body.

4. Since the electrodes and the implant had a much larger conductivity than the surrounding tissues, it was assumed that the implant (cathode) was at a constant potential, likewise the surface electrodes were modeled with a constant potential difference from the percutaneous implant. 
5. To evaluate the efficacy of electrode configuration and sizing, patient specific models were developed and the electrical potential around the implant interface was used to determine localized field strengths.

6. The model was generated using a hexahedral mesh that consisted of approximately 1.8 million elements which were treated as piecewise homogenous, ohmic and isotropic.

7. The optimal model for this experiment was selected with a relative difference $<5 \%$ in voltage gradients confirmed with a mesh sensitivity study to insure model accuracy (Table 2).

\begin{tabular}{|l|l|l|l|}
\hline \multicolumn{2}{|l|}{ Table 2 } & & \\
\hline \multicolumn{2}{|l|}{ Mesh Sensitivity Study for Amputee Model } & Relative Difference \\
\hline Mesh & Elements & 161131 & 0.0995 \\
\hline 10010050 & 149089 & 371472 & 0.0802 \\
\hline 12512575 & 350180 & 706082 & 0.0545 \\
\hline 150150100 & 673032 & 1194044 & 0.0527 \\
\hline 175175125 & 1146778 & 1860772 & 0.0439 \\
\hline 200200150 & 1796690 & 3850202 & 0.0364 \\
\hline 250250200 & 3745038 & 5226587 & 0.0301 \\
\hline 275275225 & 5097243 & 6898729 & 0.0000 \\
\hline 300300250 & 6742588 & & \\
\hline
\end{tabular}

8. Using an iterative solver, the electric metrics in the finite element models were computed for the electrode configurations.

\section{Understanding the Electrical Stimulation Paradigm}

Enhancements in medical care and evacuation strategies on the field of combat have lead to an increased number of warriors surviving disastrous war related injuries. While the improved survival rate is a medical advancement, servicemen and women are returning from combat with amputations that require intensive follow-up care, extensive rehabilitation and expensive prosthetic services from the Veteran Affairs Health Care System ${ }^{3}$. Congressional reports detail that over 1,000 war-related amputations have occurred as a result of Operation Enduring Freedom $(\mathrm{OEF})$ and Operation Iraqi Freedom (OIF) conflicts ${ }^{4}$

In the case of OEF and OIF veterans, approximately $15 \%$ of returning warriors have lost multiple limbs and a significant number of returning servicemen and women have short residual limbs where socket technology is not an option or has been rejected by the patient. The reported discontinued use of upper extremity prosthesis even exceeds $50 \%$ because the fixation devices are cumbersome and difficult to use comfortably ${ }^{5}$. Lower extremity prostheses are equally problematic and the common problems associated with soft tissue sockets include the inability to walk on challenging terrain6, limited residual limb length ${ }^{7}$, patient discomfort ${ }^{5}$, concern with non-physiological loading ${ }^{8}$, irritation from heterotopic ossification ${ }^{9}$ and risk of debilitating disease ${ }^{10}$. However, osseointegration technology is a novel surgical technique that may reduce pain $^{11}$, skin irritation ${ }^{12}$, enhance osseoperception ${ }^{13}$, improve mobility ${ }^{6}$, decrease pressure sores associated with sockets ${ }^{6}$, reduce energy for ambulation ${ }^{7,14}$ and better serve veteran and warriors with limited residual limb length ${ }^{15}$.

Despite the numerous physical and psychological advantages of osseointegration, the associated surgical procedures require more advanced infection prevention treatment streategies ${ }^{16}$, require long rehabilitation programs and include restrictive weight bearing protocols which may last up to 1.5 years postoperative ${ }^{17}$. Because the viability of the host bone and length of the residual limb is important for muscle attachment and functionality, developing new devices to improve osseointegration is key for returning servicemen and women. Therefore, development of an osseointegrated intelligent implant design (OIID) system which uses controlled electrical stimulation may reduce the length of rehabilitation and increase skeletal attachment for veteran and warrior amputees. However, since no current device is commercially available and directed for use with percutaneous osseointegrated implants, the motivation of the program is to confirm safety and efficacy with finite element analysis.

Understanding the role of electrical stimulation in bone remodeling, specifically the deposition of osteoids and mineralization, has remained speculative. However, electrical activity observed in bone may be the result of mechanical loading ${ }^{18,19}$ and therefore an electrical stimulus may be an effective mechanism for inducing bone repair ${ }^{19}$. The logic behind the hypothesis is explained in a fracture healing model. When long bones are loaded, the side in tension becomes electropositive and the compression side electronegative ${ }^{20,21}$, however, once a bone is broken, the site will remain electronegative with respect to the surrounding environment until healing has commenced and homeostasis resumed ${ }^{21}$. Simulating the natural healing cascade with an electrical signal has been believed to assist with calcium deposition ${ }^{22}$, slight alterations in oxygen content and $\mathrm{pH}^{23}$, recruitment of growth factors ${ }^{22}$ and assists with osteoblast migration and secretion of additional extracellular matrix ${ }^{24}$.

The premise that electrical stimulation alone could govern complete bone repair has been redefined and the current new hypothesis proposes that complete unions are formed by mechanical loads and an electrical stimulation co-stimulus ${ }^{19}$. The electrical impulses observed in vivo are associated with piezoelectric deformation of collagen or the large electro-kinetic currents produced by ionic constituents flowing past mineral portions of the bone matrix ${ }^{25}$. In fact, spontaneous potentials have been reported in bone as large as 6 millivolts and correlates with an increase in the mineral apposition rate of bone ${ }^{26}$.

Early work by Brighton and Friedenberg ${ }^{18,21,27,28}$ used the concept of electrical stimulation for bone regeneration in the 1960s and 1970s and demonstrated that direct current could be used to repair non-unions in a shorter period of time when compared to traditional healing methods. 
Additional models have investigated bone formation with restrictive weight bearing and demonstrated a thirty-one percent increase in osteogenic activity between controls and electrically stimulated limbs ${ }^{25}$.

While researchers in the field of electrical stimulation have paved the way for understanding the mechanism for osteoblast matrix deposition with electrical stimulation, inadequate understanding has limited the expansion of this technology. While there are many cases of successful healing of non-unions and fracture healing models, examples of patient discomfort and failed attempts are replete in the literature as well ${ }^{29}$. The problem with electrical stimulation occurs from scientists and clinicians controlling the wrong electric metrics and concentrating solely on current magnitudes. Previous researchers have looked to current as the "magic bullet" to fixing the approximate 500,000 non-unions which occur annually ${ }^{30}$. However, repeatability between models has been limited from joule heating complications ${ }^{31}$ and not determining current densities $^{32}$. In fact, all manufactured biomedical devices must be limited to a current density less than $2 \mathrm{~mA} / \mathrm{cm}^{2}$ as outlined by the International Electrotechnical Commission to prevent localized tissue necrosis and patient discomfort ${ }^{33}$.

Aside from assisting with skeletal fixation, controlled electrical stimulation may also prevent bacterial adhesion on orthopedic implants and reduce the risk for osteomyelitis and biofilm formation ${ }^{34-37}$. Biofilm formation on orthopedic devices lead to patient complications and significant distress for those who depend on these devices ${ }^{38}$. Emphasis is placed on the necessity to have completely sterilized instrumentation and implants prior to surgery ${ }^{39}$, however it is often difficult to diagnose bacteria adhesion as evident from many negative cultured cases which indeed are infected ${ }^{40}$. This problem is often coupled with the fact that biofilms are slow growing in nature ${ }^{40}$, cannot be growth accurately in vitro ${ }^{39}$, depend on the type of bacteria cells, cleanliness of the surface and the immune system of the affected person ${ }^{39}$. Investigation of European transfemoral amputees with oseeointegration technology reveal the most frequent problem is infection (frequent superficial infections, $1 / 3$ periprosthetic infections) ${ }^{41}$. While there have been vast improvements in surgical preparation, eradicating bacteria is one of the fundamental factors for improving osseointegration since biofilms are between 500-5000 thousand times more difficult to eradicate due to their non-platonic form ${ }^{34,35,39}$. Therefore, utilizing electrical stimulation as a modality for removing harmful bacterial colonies and increasing skeletal fixation are important factors for ensuring the protection of patient health and OIID efficacy.

The advantages of using veteran and warrior amputees are that the relative youth and otherwise good health of these individuals make them an ideal population for aggressive rehabilitation and a percutaneous post will serve as an ambulatory aid and may be developed as an exposed cathode for electrical stimulation. The presence of an osseointegrated implant does not require additional surgical procedures to insert electrical components, allows the device to be controlled externally and prevents further risk of infection ${ }^{42}$. Therefore, by understanding the method of current injection into the residual limb of veteran and warrior amputees, an electric field on the magnitude of $1-10 \mathrm{~V} / \mathrm{cm}$ may be established, controlled and measured at the implant interface. It is hypothesized that this will allow safe levels of electricity to be delivered, capable of inducing osteoblast migration and improving skeletal attachment. An electric field of this degree will increase the quantity and quality of bone at the implant interface, and improve the prospects for accelerated rehabilitation and skeletal fixation for an amputee. Use of electrical stimulation has not been investigated as a modality to accelerate osseointegration in an intramedullary prosthetic implant and presents numerous opportunities for translational research to improve patient care.

\section{Experimental Results}

The necessity for patient specific models with a percutaneous electrical stimulation device was supported in the study. The simulations developed for the proposed biomedical device may have the capabilities of expediting skeletal attachment by increasing osteoblast migration and preventing bacterial adhesion $27,34,36,39$. Computation modeling has effectively shown that $1-10 \mathrm{~V} / \mathrm{cm}$ electric fields and current densities below $2 \mathrm{~mA} / \mathrm{cm}^{2}$ may be generated using the implant as a functional cathode and is most homogenously distributed using a two band external electrode. The OIID system may be the first step to resolving the classic problem associated with electrical stimulation; the inability to define current pathways in the human body ${ }^{43}$. Therefore, establishing tools for enhancing skeletal attachment may assist with reducing the length of rehabilitation required for an osseointegrated procedure.

Utilizing electrical stimulation for older amputees is also a critical aspect which must be explored as well. Bone mass is maximum a decade after skeletal growth ceases but decreases significantly by the eighth and ninth decade ${ }^{44}$. As long bones change with age, the endosteal diameter tends to increase more rapidly than the periosteal diameter which may lead to implant loosening ${ }^{45}$. This problem coupled with the reduction of strain on bones by weaker muscles may contribute to debilitating diseases such as osteoporosis and osteopenia ${ }^{45}$ and require additional treatment options for patients with osseointegrated implants. However, controlled electrical stimulation and mechanical loading may act as a synergistic catalyst of bone ongrowth and maintain host bone bed integrity with elderly patients using an OIID system.

\section{Disclosures}

The technology disclosed in the article, specifically utilizing an orthopedic implant as a functional cathode to increase skeletal attachment, reduce rehabilitation and prevent bacterial adhesion is a novel idea invented by the first author in the journal submission. An invention disclosure form has been submitted to the University of Utah and a United States provisional patent has been filed protecting all technology disclosed.

\section{Acknowledgements}

This material is based upon research supported (or supported in part by) the Technology Commercialization Office, Salt Lake City, UT, Office of Research and Development, Rehabilitation R\&D Service, DVA SLC Health Care System, Salt Lake City, UT, Department of Defense PRMRP Grant (No. PR054520), the Albert \& Margaret Hofmann Chair and the Department of Orthopaedics, University of Utah School of Medicine, Salt Lake City, UT; Technical support for the simulations was provided by the Center for Integrative Biomedical Computing of Scientific Computing and Imaging Institute and was made possible in part by software from the NIH/NCRR Center for Integrative Biomedical Computing, P41RR12553-07. 
Additional gratitude is extended to Gwenevere Shaw for assistance with manuscript preparation and Dustin Williams for the image of biofilm.

\section{References}

1. Tortora, G.J., and Nielsen, M.T., in Principles of Human Anatomy, edited by B. Roesch et al. John Wiley \& Sons, Inc., Hoboken, NJ, 117 (2009).

2. Bloebaum, R.D., Bachus, K.N., Momberger, N.G., and Hofmann, A.A. Presented at the Orthopaedic Research Society 39th Annual Meeting Trans. Orthopaed. Res. Soc., San Francisco, CA, 1993 (unpublished); Bloebaum, R.D., Bachus, K.N., Momberger, N.G., and Hofmann, A.A. Journal of Biomedical Materials Research. 28 (5), 537 (1994).

3. Goldberg, M.S. Military Medical/NBC Technology. 11 (8), 31 (2007); Kerr, J.C. in The Salt Lake Tribune. Salt Lake City (2008).

4. Fischer, H. Report No. Order Code RS22452, (2008).

5. Moore, T.J., et al. Clin Orthop Relat Res. 238, 219 (1989).

6. Hagberg, K., and Branemark, R. Prosthet Orthot Int. 25 (3), 186 (2001).

7. Todd, T.W., and Barber, C.G. J Bone Joint Surg Am. 16, 53 (1934).

8. Jaegers, S.M., Arendzen, J.H., and de Jongh, H.J. Archives of Physical Medicine and Rehabilitation. 76 (8), 736 (1995); Jaegers, S.M., Arendzen, J.H., and de Jongh, H.J. Clin Orthop Relat Res. 319, 276 (1995).

9. Potter, B.K., et al. Journal of American Academy of Orthopaedic Surgeons. 14 (10 Suppl), S191 (2006)

10. Kulkarni, J., Adams, J., Thomas, E., and Silman, A. Clin Rehabil. 12 (4), 348 (1998).

11. Smith, D.G., et al. Clin Orthop Relat Res. (361), 29 (1999).

12. Pasquina, P.F., et al. Archives of Physical Medicine and Rehabilitation. 87 (3 Suppl 1), S34 (2006); Meulenbelt, H.E., Geertzen, J.H., Jonkman, M.F., and Dijkstra, P.U. Archives of Physical Medicine and Rehabilitation. 90 (1), 74 (2009).

13. Ysander, M., Branemark, R., Olmarker, K., and Myers, R.R. Journal of Rehabilitation Research \& Development. 38 (2), 183 (2001); Jacobs R., and Van Steenberghe, D. Journal of Oral Rehabilitation. 33 (4), 282 (2006).

14. Couch, N.P., David, J.K., Tilney, N.L., and Crane, C. American Journal of Surgery. 133 (4), 469 (1977); Huang, C.T., et al. Archives of Physical Medicine and Rehabilitation. 60 (1), 18 (1979); Waters, R. L., and Lunsford, B.R. in Atlas of Orthotics. edited by C. V. Mosby, St. Louis, MO, 151 (1985); Waters, R.L., Perry, J., Antonelli, D., and Hislop, H. J Bone Joint Surg Am. 58 (1), 42 (1976).

15. Morgenroth, D.C., Shakir, A., Orendurff, M.S., and Czerniecki, J.M. Am J Phys Med Rehabil. 88 (2), 108 (2009).

16. Pendegrass, C.J., et al. Journal of Bone and Joint Surgery British. 90 (1), 114 (2008); Pendegrass, C. J., Goodship, A. E., and Blunn, G. W., Biomaterials. 27 (23), 4183 (2006).

17. Lee, W.C., et al. Med Eng Phys. 30 (7), 825 (2008).

18. Brighton, C.T., Friedenberg, Z.B., Zemsky, L.M., and Pollis, P.R. J Bone Joint Surg Am. 57 (3), 368 (1975).

19. Spadaro, J.A. Bioelectromagnetics. 18 (3), 193 (1997).

20. Brighton, C.T., Friedenberg, Z.B., Mitchell, E.I., and Booth, R.E. Clin Orthop Relat Res. 124, 2 (1976).

21. Friedenberg, Z.B., and Brighton, C.T., J Bone Joint Surg Am. 48 (5), 915 (1966).

22. Yonemori, K., et al. Bone. 19 (2), 173 (1996).

23. Treharne, R.W., Brighton, C.T., Korostoff, E., and Pollack, S.R. Clin Orthop Relat Res. (145), 300 (1979); Matsunaga, S. J. Jpn. Orthop. Ass. 60 (12), 1293 (1986); Robblee, L.S., and Rose, T.L. Neural prostheses : Fundamental Studies. Prenctice Hall, Upper Saddle River (1990).

24. Wiesmann, H., et al. Biochimica et Biophysica Acta. 1538 (1), 28 (2001); Ferrier, J., Ross, S.M., Kanehisa, J., and Aubin, J.E. Journal of Cellular Physiology. 129 (3), 283 (1986)

25. McLeod, K.J., and Rubin, C.T. J Bone Joint Surg Am. 74 (6), 920 (1992).

26. Rubinacci, A., and Tessari, L. Calcified Tissue International. 35 (6), 728 (1983).

27. Brighton, C.T. J Bone Joint Surg Am. 63 (5), 847 (1981); Friedenberg, Z.B., et al. Surgery, Gynecology \& Obstetrics. 131 (5), 894 (1970).

28. Friedenberg, Z.B., Zemsky, L.M., Pollis, R.P., and Brighton, C.T. J Bone Joint Surg Am. 56 (5), 1023 (1974).

29. Jorgensen, T.E. Clin Orthop Relat Res. 124, 124 (1977); Minkin, C., Poulton, B.R., and Hoover, W.H. Clin Orthop Relat Res. 57, 303 (1968).

30. Ehrlich, G.D., et al. Clin Orthop Relat Res. 437, 59 (2005).

31. Soong, H.K., et al. Investigative Ophthalmology \& Visual Science. 31 (11), 2278 (1990).

32. Li, W.P., et al. Burns. 32 (8), 986 (2006).

33. Leitgeb, N., Cech, R., and Schrottner, J. Radiat Prot Dosimetry. 124 (2), 124 (2007).

34. van der Borden, A.J., et al. Biomaterials. 28 (12), 2122 (2007); van der Borden, A.J., van der Werf, H., van der Mei, H.C., and Busscher, H.J. Applied and Environmental Microbiology. 70 (11), 6871 (2004).

35. van der Borden, A.J., van der Mei, H.C., and Busscher, H.J. Biomaterials. 26 (33), 6731 (2005).

36. Costerton, J.W., et al. Annual Review of Microbiology. 41, 435 (1987); Costerton, J.W., et al. Antimicrobial Agents and Chemotherapy. 38 (12), 2803 (1994).

37. Neut, D., van der Mei, H.C., Bulstra, S.K., and Busscher, H.J. Acta Orthop. 78 (3), 299 (2007); O'Gara, J.P., and Humphreys, H. Journal of Medical Microbiology. 50 (7), 582 (2001); Blenkinsopp, S.A., Khoury, A.E., and Costerton, J.W. Applied and Environmental Microbiology. 58 (11), 3770 (1992).

38. Anwar, H., Dasgupta, M.K., and Costerton, J.W. Antimicrobial Agents and Chemotherapy. 34 (11), 2043 (1990).

39. Costerton, J.W. Clin Orthop Relat Res. (437), 7 (2005).

40. Nelson, C.L., et al. Clin Orthop Relat Res. 437, 25 (2005).

41. Gunterberg, B., et al. presented at the Conference Book of IXth World Congress ISPO (unpublished). Amsterdam, The Netherlands (1998).

42. Lavine, L.S., and Grodzinsky, A.J. J Bone Joint Surg Am. 69 (4), 626 (1987).

43. Chakkalakal, D.A., and Johnson, M.W. Clin Orthop Relat Res. (161), 133 (1981); Noda, M., and Sato, A. Clin Orthop Relat Res. (193), 288 (1985).

44. Buckwalter, J.A., Glimcher, M.J., Cooper, R.R., and Recker, R. J Bone Joint Surg Am. 77 (2), 1276 (1995).

45. Lane, J.M., and Vigorita, V.J. J Bone Joint Surg Am. 65 (2), 274 (1983). 